\title{
Pseudomyxoma Peritonei As A Result Of Low Grade Appendiceal Mucinous Neoplasm In Association With Ovarian Fibroma.
}

\author{
Dr.Deepa.R ${ }^{1}$, Dr.R.Sathyalakshmi ${ }^{2}$, Dr. Nalli. R . Sumitra Devi ${ }^{3}$, \\ Dr. R. Padmavathi ${ }^{4}$, Dr.Mary Lilly. $\mathrm{S}^{5}$ \\ Department of Pathology, Stanley Medical College, Chennai, Indial. \\ Department of Pathology, Stanley Medical College, Chennai, India ${ }^{2}$. \\ Department of Pathology, Stanley Medical College, Chennai, India ${ }^{3}$ \\ Department of Pathology, Stanley Medical College, Chennai, India ${ }^{4}$. \\ Department of Pathology, Stanley Medical College, Chennai, India ${ }^{5}$
}

\begin{abstract}
Context : Pseudomyxoma peritonei is a condition caused by the production of abundant mucin by tumor cells which fills the abdominal cavity.(1)The tumor causes fibrosis of tissues and impedes digestion and organ function. If left untreated, tumor and mucin can build up to the point where it compresses vital structures: the colon, the liver, kidneys, stomach, spleen, etc .This disease is most commonly caused by an appendiceal primary tumor.

Aims : We describe a case of a 65 year old female with pseudomyxoma peritonei as a result of low grade appendiceal mисіnous neoplasm in association with ovarian fibroma. No such cases have been reported in literature till date.

Methods and Materials : The patient underwent Left ovariectomy with appendicectomy, caecal perforation closure and diverting loop ileostomy.

Conclusion: Thus we report a rare occurrence of pseudomyxoma peritonei as a result of low grade appendiceal mucinous neoplasm and ovarian fibroma.
\end{abstract}

Key words : Mucinous neoplasm, appendix, ovarian fibroma

\section{Introduction}

Mucinous neoplasms of the appendix are rare tumors, some of them are characterized by a questionable discrepancy between a benign morphologic appearance and an aggressive biologic potential that can be associated with poor prognosis and high mortality. The clinical picture of pseudomyxoma peritonei is, with few exceptions, caused by mucinous appendiceal neoplasms and differs in many aspects from the usual peritoneal carcinomatosis. The controversy regarding terminology, diagnostic criteria, classification and therapy of these tumors has lasted for decades. The revised edition of the World Health Organization Classification of Tumors of the Digestive System proposes a uniform reporting system for mucinous appendiceal neoplasms and the peritoneal disease associated with it, thereby creating a comparable basis for pathological diagnosis, clinical therapy and further scientific studies.(2) Here we have described one such case of pseudomyxoma peritonei associated with ovarian fibroma.

\section{Case Report}

A 65 year old female presented with abdominal mass for four days associated with fever. On examination of the abdomen there was a vague mass in the right iliac fossa with minimal guarding. Blood investigations showed that the patient was anemic. Ultrasound examination of the abdomen showed heterogenous hypoechoic mass lesion in fundus of the uterus,ascites, hypoperistalitic small bowel loop and. ileaocaecal thickening . CT abdomen showed well defined enhancing soft tissue lesion in left adnexa, moderate ascites, thickened omentum and mild thickening of parietal peritoneum. After being diagnosed as a case of intestinal obstruction the patient underwent emergency laparotomy . Intra operative findings were as follows

\section{Findings: -}

1. $10 \times 8 \mathrm{~cm}$ tumour arising from left ovary which was hard in consistency causing compression of sigmoid colon leading to bowel obstruction

2. Dilated descending colon/ Transverse Colon / Ascending colon with thickening of ceacum.

3. Gangrenous appendix with thickening of cecum causing smalI intestinal obstruction.

4. Multiple mucinous jelly all over the peritoneal cavity.

5. Multiple flakes over the small intestine/large intestine. 
6. Purulent collection in the pelvis.

7. Liver,gall bladder, spleen, - normal.

8. No free fluid.

The treatment given was Left ovariectomy with appendicectomy, caecal perforation closure and diverting loop ileostomy. The specimen of ovary and appendix was sent for histopathological examination.

On gross examination ovary measured $8 \times 6 \times 3 \mathrm{cms}$. External surface was smooth and nodular. Cut surface was grey white, firm, homogeneous and glistening. Appendix was received as single whitish soft tissue with central lumen measuring 4 × 4 × $1 \mathrm{cms}$ surrounded by whitish gelatinous soft tissue.

Microscopic picture :

Sections from the appendix showed a neoplasm arranged in cystoglandular and papillary pattern lined by columnar epithelial cells showing nuclear stratification with moderate nuclear atypia. Lumen was filled with pools of mucin and strands of epithelium infiltrating fibromuscular tissue of appendix.

Sections from the ovary showed a well circumscribed neoplasm composed of spindle shaped cells arranged in fascicles and interlacing bundles separated by extensive bands of hyalinization. The tumor cells were spindle shaped with moderate cytoplasm and spindle shaped nuclei with mild atypia. The diagnosis of benign fibroma with extensive hyalinization was reached.

\section{Discussion}

In this case we report a rare association of pseudomyxoma peritonei due to mucinous neoplasm of appendix with fibroma of the ovary. Literarily translated pseudomyxoma peritonei means false mucinous tumor. Mucocele of the appendix was recognized as a pathologic entity by Rokitansky in 1842 and was formally named by Feren in 1876 (3), but it was R. Werth (4) who used the term "pseudomyxoma peritonei" for the first time in 1884 when he described its occurrence in association with a mucinous carcinoma of the ovary. The incidence of pseudomyxoma peritonei is approximately two in 10,000 laparotomies, and about $75 \%$ of patients are female with an average age of 53 years .(5)

In a large majority of patients (80\%), pseudomyxoma peritonei arises from an appendicular disease and not ovarian disease .(6). Appendicael type mucinous tumor in an ovarian teratoma can result in pseudomyxoma peritonei.(14) The most common sites of origin are considered the appendix and the ovary, although other sites, such as the gallbladder,stomach, pancreas, colon, uterus, fallopian tubes, urinary bladder, breast and lung, have also been described. $(7,8)$

Because of the rarity of this condition, the diagnosis of pseudomyxoma peritonei is often difficult.

Ultrasonographic findings are echogenic ascites with multiple semi-solid tumors and scalloping of the hepatic and splenic margins from external pressure of adjacent

peritoneal implants .(9)

On CT, pseudomyxoma peritonei is characterized by low-attenuation mucinous ascites, associated with scalloping of the liver margins and hypodense peritoneal implants associated with bowel implants.(10). Microscopically the cells are arrangedin small strips, groups, acinar structures and singly often with abundant intracellular mucin. The cytologic dysplasia may be subtle. The cells generally express CD-X2 and Ck 20 and there is co-expression of Ck 7.The goblet cells are found to express MUC 2 and MUC 5AC.(15).Pseudomyxoma peritonei is classified as low grade and high grade. In low grade the epithelium forms $<10 \%$ of the tumor cells ther is mild cytological atypia with few mitoses . thw high grade variety shows high cellularity with desmoplastic stroma, moderate to severe cytological atypia with enlarged nucleoli and numerous mitoses.(14). The differential diagnoses include extensive myxoid change in endometriosis and myxoid mesenchymal lesions like myxoid leiomyosarcoma and aggressive angiomyxoma.(14).

Ovarian fibroma is a stromal tumor, that accounts for $4 \%$ of all ovarian tumors. It usually occurs in patients over the age of 40 years. Ovarian fibromas usually present as unilateral, solid, hard masses with a bosselated external surface. Edematous tumors are usually soft in consistency and cyst formation is common. The cut surface is grey-white and homogeneous with a whorled pattern with occasional areas of calcification. It may be associated with ascites and hydrothorax known as Meig's Syndrome.Some association with Gorlins syndrome has also been reported. But so far no association of ovarian fibroma with pseudomyxoma peritonei due to mucinous appendiceal neoplasm has been reported.(11)

Ovarian fibromas are stromal tumors composed of spindle, oval or round cells producing collagen.There are areas of hyaline plaques, edema and increased vascularity. Fibromas are solid, spherical, slightly lobulated, encapsulated, grey-white masses covered by a glistening, intact ovarian serosa.Fibromas occur at all ages, most frequently during middle age, with an average age of 48 years.Ovarian fibromas are almost always benign. Very rarely, fibromas without any atypical features are associated with peritoneal implants(12).Grossly the differential diagnoses includes krukenberg's tumor, Brenner and carcinoid tumor. Microscopically the differentials includes fibromatosis that envelopes the follicles rather than displacing them 
like fibroma and tumors with cystic degeneration.(15). Surgical removal of these solid ovarian tumors is recommended because of the low probability of malignancy(13).

Thus here we describe a rare association of appendiceal mucinous neoplasm with fibroma of the ovary.

\section{References}

[1]. Qu Z, Liu L."Management of pseudomyxoma peritonei". World J Gastroenterol .2006;12 (38): 6124-7.

[2]. Mucinous neoplasms of the vermiform appendix, Pseudomyxoma peritonei, and the new WHO classification.Pathologe. 2012 Feb;33(1):24-30

[3]. de Rezende Pereira JC, Trugilho JC, Sarmat AA. Mucocele of the appendix. Surgery. 2004; 136(5): 1096-1097.

[4]. Hinson FL, Ambrose NS. Pseudomyxoma peritonei. Br J Surg.1998; 85(10): 1332-1339.

[5]. Mann WJ, Jr., Wagner J, Chumas J, Chalas E. The management of pseudomyxoma peritonei. Cancer.1990; 66(7): 1636-1640.

[6]. Elias D, Sabourin JC. Les pseudomyxomes péritonéaux. Mise au point. J Chir (Paris).1999; 136(6): 341-347.

[7]. Sherer DM, Abulafia O, Eliakim R. Pseudomyxoma peritonei: a review of current literature. Gynecol Obstet Invest. 2001; 51(2): 73-80. Shimoyama S, Kuramoto S, Kawahara M, Yamasaki

[8]. $\mathrm{K}$, Endo $\mathrm{H}$, Murakami $\mathrm{T}$, et al. A rare case of pseudomyxoma peritonei presenting an unusual inguinal hernia and splenic metastasis. J Gastroenterol Hepatol.2001; 16(7): 825-829.

[9]. Lersch C, Frimberger E, Ott R,Classen M. Gray-scale sonographic findings in a patient with pseudomyxoma peritonei. J Clin Ultrasound. 2001; 29(3): 186-191.

[10]. Sulkin TV, O’Neill H, Amin AI, Moran B. CT in pseudomyxoma peritonei: a review of 17 cases. Clin Radiol.2002; 57(7): 608613.

[11]. Arya A, Rao S, Agarwal S, Arora R, Gupta K, Dhawan I. Ovarian fibroma: An unusual morphological presentation with elevated CA-125. Indian J Pathol Microbiol [serial online] 2008 [cited 2013 Oct 7];51:523-4.

[12]. Young RH, Scully RE. Sex Cord-Stromal, Steroid Cell and other ovarian tumors with Endocrine, Paraendocrine and Paraneoplastic manifestation. In: Kurman RJ, editors. Blausteins Pathology of the Female Genital Tract. $5^{\text {th }}$ ed. India: Springer Private Limited. 2004: 923-5.

[13]. Leung SW, Yuen PM. Ovarian fibroma: A review on the clinical characteristics, diagnostic difficulties and management option in 23 cases. Gynecol Obstet Invest .2006;62:1-6.

[14]. Robert . D. Odze, John . R. Goldblum. Surgical pathology of GI tract, Liver , Biliary tract and Pancreas, second edition.2009:64548.

[15]. Sternberg's Diagnostic Surgical Pathology, Fifth Edition.2010;2:2353.

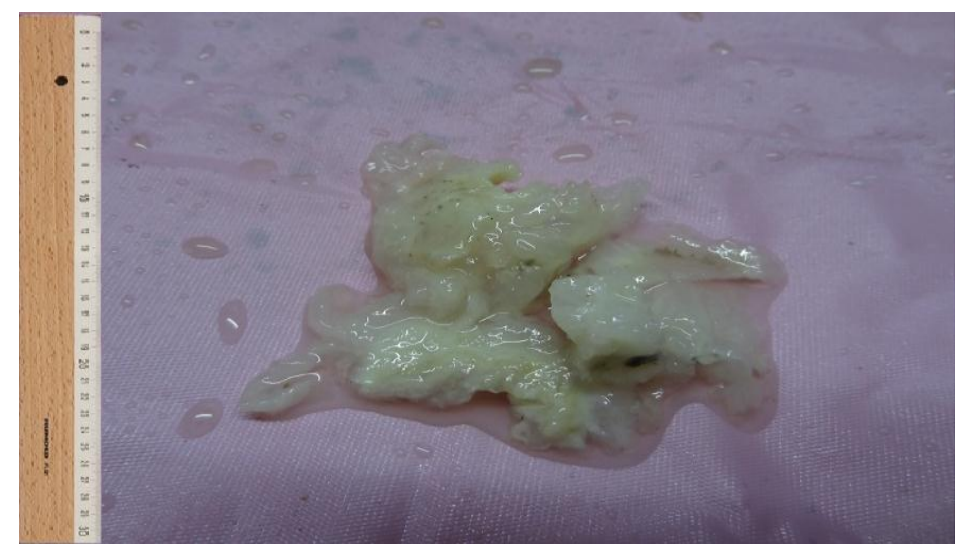

Fig 1 : gross picture of appendix with its lumen and surrounded by gelatinous material.

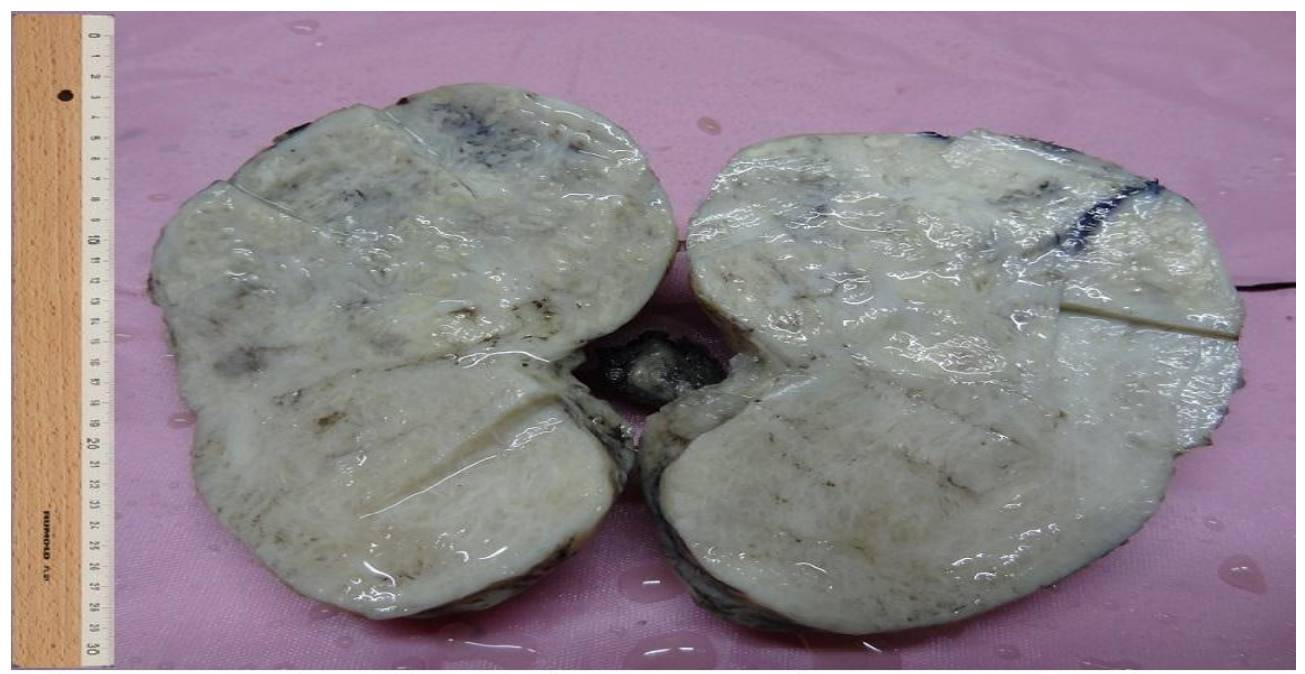

Fig 2: gross picture of the ovary showing fibrosis. 


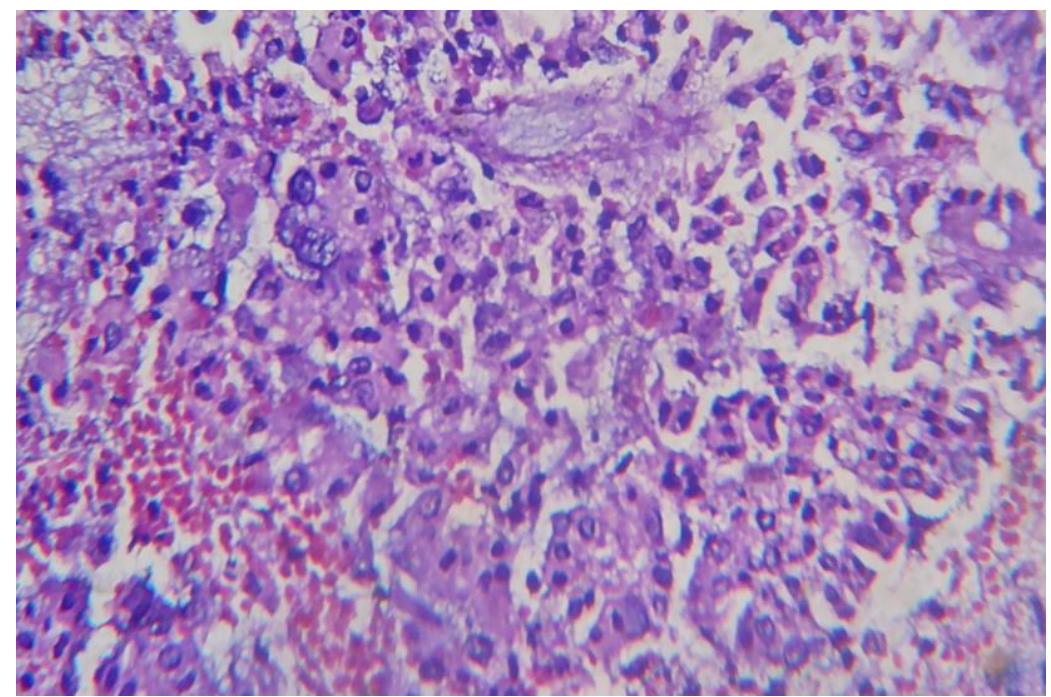

Fig 3: microscopic picture of the appendix showing mucinous areas

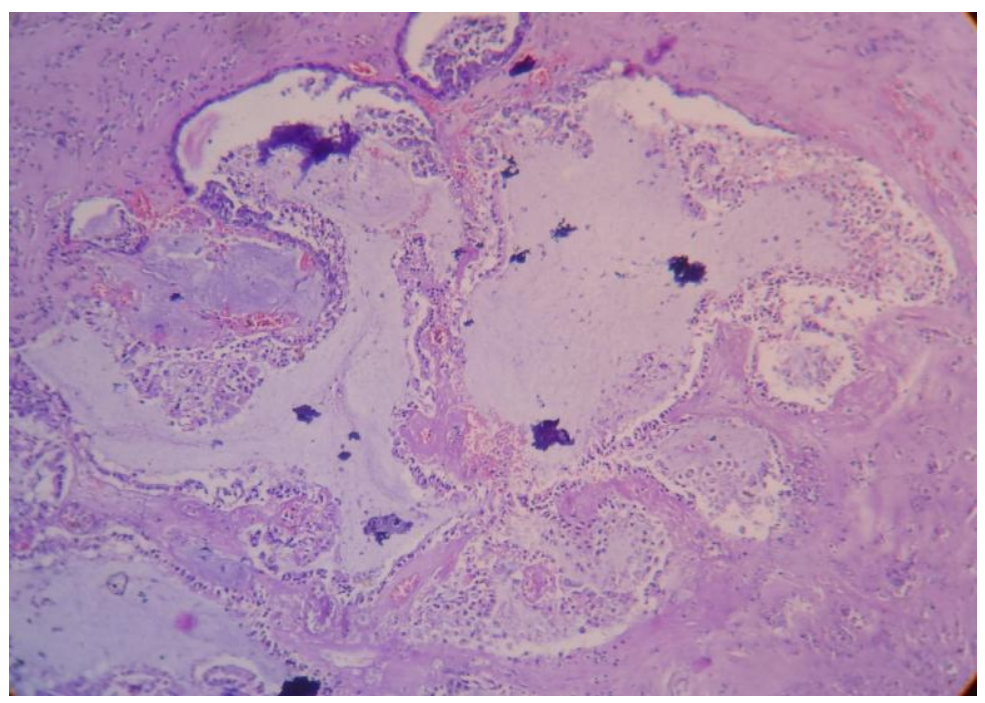

Fig 4 : microscopic picture of the appendix showing mucin filled lakes.

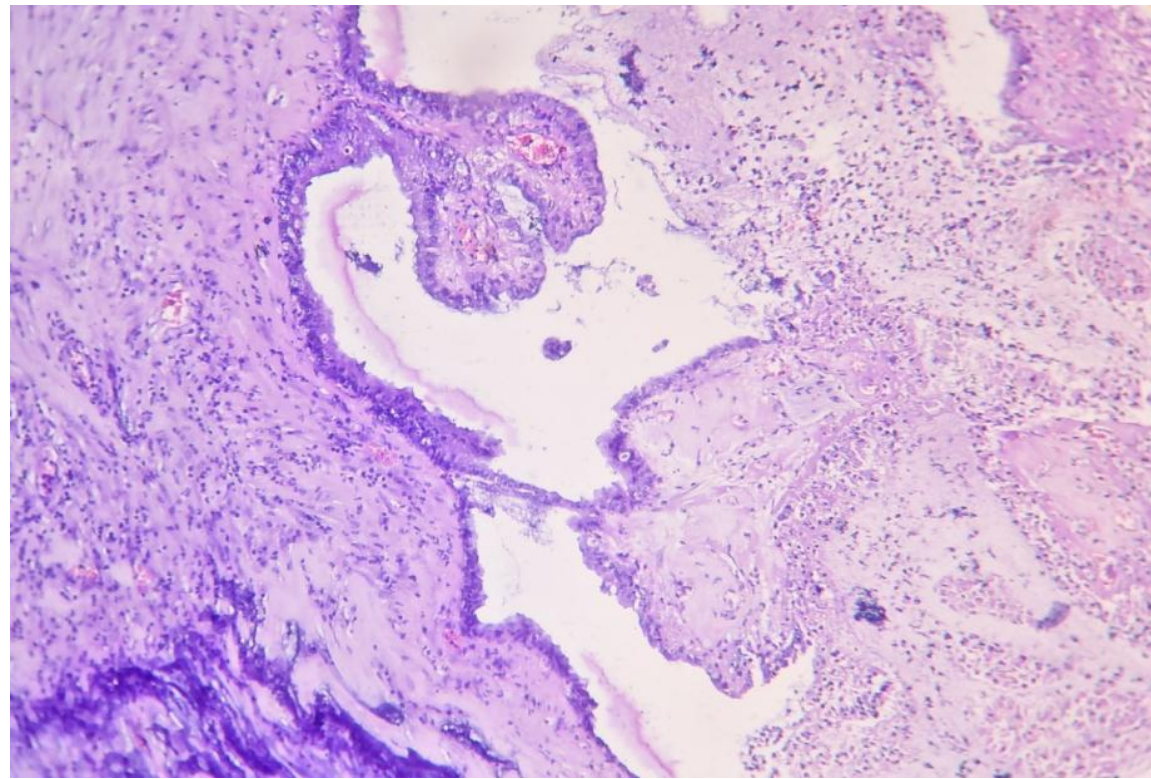

Fig 5 : microscopic picture of the appendix showing papillary area lined by mucinous cells and with a vascular core. 


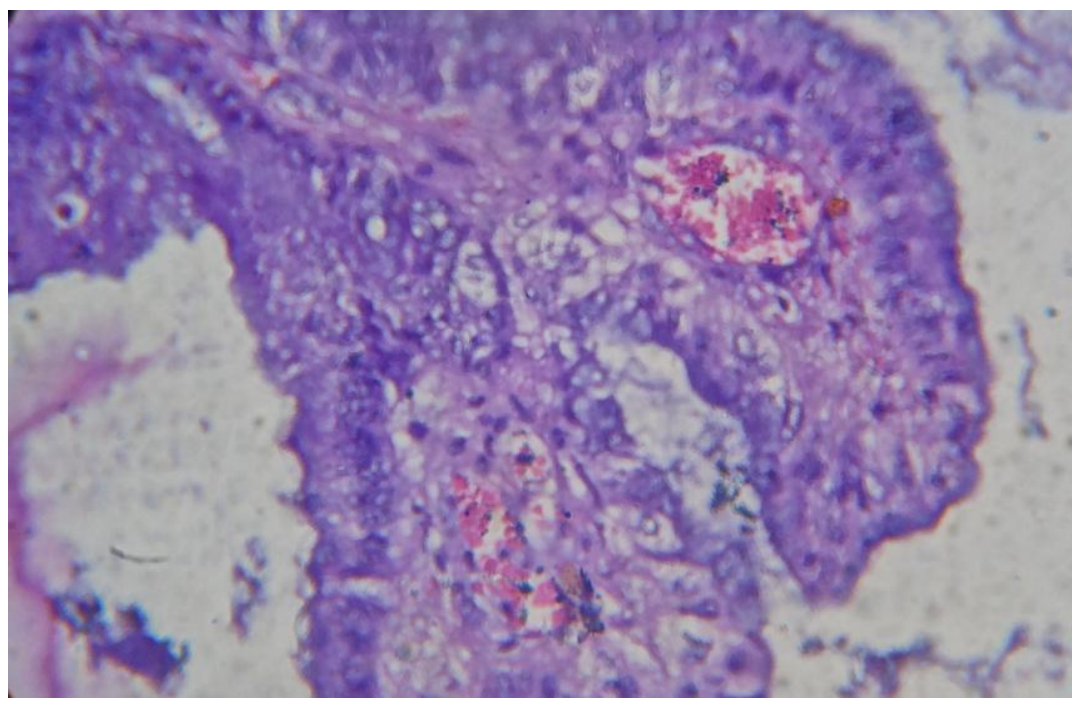

Fig 6 : high power picture of the papillary area.

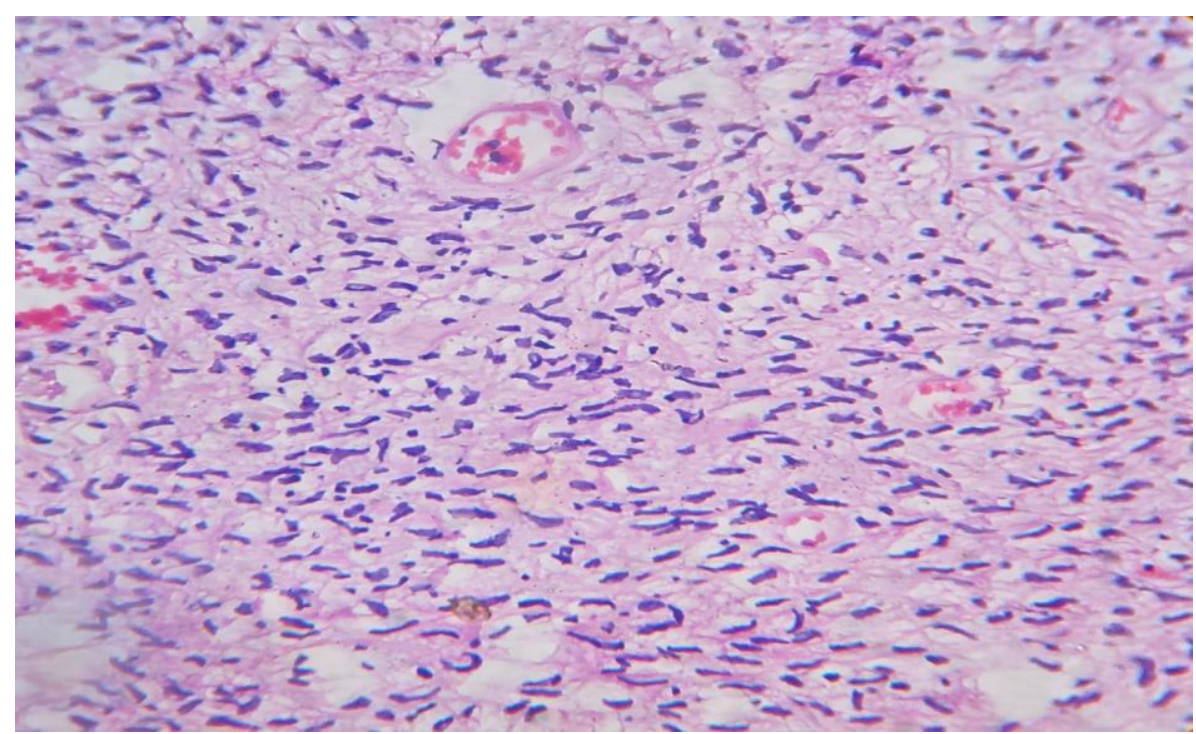

Fig 7 : microscopic picture of ovarian fibroma. 